\title{
EDITORIAL
}

\section{Emerging novel approaches to drug research and diagnosis of Parkinson's disease}

\author{
Acta Pharmacologica Sinica (2020) 41:439-441; \\ https://doi.org/10.1038/s41401-020-0369-7
}

Parkinson's disease (PD) is one of the most common neurodegenerative diseases. Genetic factors are involved in $<10 \%$ of PD cases, while the majority of PD cases have no identifiable genetic factors. All these studies have revealed the heterogeneity of Parkinson's disease both in pathogenesis and etiology [1, 2]. In the past decade, many well-designed longitudinal studies have clearly elucidated that environmental or behavioral factors can modify the risk of developing Parkinson's disease and alter the pathological process of PD [2]. Moreover, many factors that were found to be associated with an altered risk of PD in epidemiological studies have been confirmed to be neuroprotective or neurotoxic to dopaminergic (DA) neurons in experimental animal models of PD.

The progress in the etiologic study of PD provides novel insights to explore the molecular mechanisms of PD pathology. In addition to the importance of alpha-synuclein (a-syn), mitochondrial dysfunction, autophagy activities in DA neuronal death, neuroinflammation-mediated damage, alterations in networking and the circuits of basal ganglia, and changes in non-dopaminergic systems have all been shown to contribute to either the pathological process or clinical symptoms of PD [3-5]. Although mechanistic studies have made some important discoveries, there is still no cure for PD. However, breaking through a therapeutic approach that can modify disease progression is very limited to pilot clinical studies or animal experiments $[6,7]$. In this context, dopamine replacement remains the gold standard to relieve motor symptoms [6]. In the past few years, a non-dopaminergic drug that targets the adenosine A2A receptor has been approved as an adjunct therapy for PD $[8,9]$. Given the advance in PD research, we thus edited this special issue in which we invited a number of contributors to provide wide spectral coverage on the progress in PD studies.

Neurotoxin-induced animal models have been widely used for PD pathological study and drug development $[10,11]$. Although acute neurotoxin administration can induce selective death of DA neurons, which mimics the pathology of PD, those animal models, however, fail to reproduce the progressive nature of the disease. In recent years, a variety of genetic models based on PD risk genes have also been developed and used for mechanistic studies $[12,13]$. In this special issue, Dr. Zhen's group employed DAT promoter-mediated Cre transgenic mice to establish tamoxifeninducible Dicer conditioned knockout (cKO) mice and found that upon tamoxifen administration, Dicer cKO mice manifested a progressive loss of DA neurons in the substantia nigra (SN). Robust loss of DA neurons was observed at week 6 following tamoxifen induction. They reported the detailed pathological development, including neuroinflammation alone with the deletion of Dicer. Importantly, the PD-like phenotypes in motor functions also developed progressively, which was consistent with pathological development. This study also validated the value of Dicer CKO mice for PD drug research by employing various known drugs that have been shown to produce antiparkinsonian efficacy. The results showed that the Dicer CKO mice nicely mimicked the progressive process of PD and could be a useful model to investigate the pathological development of PD and intervention-mediated changes [14].

The identification and validation of new targets that can modify the disease process aresome of the main focuses in PD research. Angiogenin is a ribonuclease with both nuclear and cytosolic activities. Multipharmacological effects of angiogenin have been reported. In addition to stimulation of endothelial cell proliferation, angiogenin increases ribosomal RNA (rRNA) transcription in the nucleus. In the cytosol, angiogenin cleaves noncoding RNAs, including transfer RNAs (tRNAs). Cleavage of tRNAs by angiogenin generates fragments termed stress-induced tRNA fragments or tiRNAs, which have been shown to inhibit ribosome assembly, protein translation, and apoptosis [15]. Alterations in angiogenin have been linked to neurodegenerative diseases, including PD [16]. Dr. Prehan summarized the functional roles and therapeutic potential of angiogenin in PD and further discussed that tRFs generated by angiogenin and other ribonucleases may deliver novel diagnostic or prognostic tools for neurodegenerative disorders [17].

The debilitating motor symptoms of PD are largely caused by decreased levels of dopamine in the basal ganglia. Over the last decades, neurophysiological and anatomical studies in patients and animal models of PD have identified a series of functional and structural changes following the loss of dopamine in the basal ganglia and related brain regions. These studies suggest that PD is a complicated neural network disorder in which abnormal intrinsic excitability and synaptic properties of neurons in the basal ganglia result in pathological oscillations (particularly the b-band between 13 and $30 \mathrm{~Hz}$ ) in the cortico-basal ganglia networks [18, 19]. The pathological rate and rhythmic pattern of neuronal activity in the cortico-basal ganglia network dramatically impair normal network function in the planning and execution of movements, thus leading to motor symptoms of PD. Dr. Chu reviewed the latest advances in our understanding of molecular, cellular, and network mechanisms that underlie pathological plasticity activity of the basal ganglia in parkinsonism. Mechanistic understanding of basal ganglia pathology in the parkinsonian state will help us to design novel symptomatic or disease-modifying strategies for PD treatment [20].

The striatum is a critical nucleus in the cortico-basal ganglia circuit and contributes to normal movement control and motor learning. The cellular and synaptic activities of striatal projection neurons are under tight control of both dopaminergic and cholinergic neuromodulation. The cholinergic interneurons (Chls) in the striatum provide the majority of cholinergic input striatal projection neurons $[21,22]$. Striatal Chls and their corresponding receptors are emerging as new promising therapeutic targets for PD. Dr. Liu has reviewed the recent exciting new development in 
striatal movement control by emphasizing the roles of the cholinergic system [23].

In addition to motor abnormalities, nonmotor symptoms are increasingly recognized in PD both in pathological development and treatment. Previous studies have shown that some nonmotor symptoms, such as depression and anxiety, could occur much earlier than motor impairments in PD patients [24]. Dr. Waddington has updated the recent development in biological mechanisms of psychotic symptoms in PD and analyzed the pathobiological challenges vis-à-vis the schizophrenia spectrum and other psychoses [25].

Neuroprotection is one of the approaches to treat PD and other neurodegenerative diseases. Numerous agents, including some antipsychotic drugs, have been shown to protect DA neurons in experimental studies. Atypical antipsychotic drugs such as clozapine have been shown to exert neuroprotection, including of DA neurons. Neuroprotection has been recognized as a part of the mechanism for the antipsychotic effects of drugs. It was believed that those antipsychotic drugs inhibited glycogen synthase kinase$3 \beta$ (GSK-3 $\beta$ ) activity by promoting the Ser9 phosphorylation of GSK$3 \beta$ and stimulated the production of brain-derived neurotrophic factor, which may underlie neuroprotection and neurogenesis [26]. In addition, modulation of microglial activation is also suggested to be an important pathway for neuroprotection of clozapine [27]. Interestingly, Dr. Liang's laboratory reported that olanzapine protected DA neurons against neurotoxicity induced by rotenone via activation of AMPK and its regulation of autophagy [28]. This finding provides a novel mechanism for the neuroprotection mediated by atypical antipsychotic drugs.

Natural products from traditional Chinese medicine or herbs are an enriched source of neuroprotective agents that have been found to prevent dopaminergic loss in various experimental PD animal models [29]. For instance, Dr. Du's group reported that ganoderic acid $A$, a triterpene found in ganoderma mushrooms, protected dopaminergic neurons from excessive nitric oxide damage, probably via modulation of $\beta$-adrenergic receptors [30].

Ginsenoside Rg1 extracted from the Panax ginseng C.A. Meyer has been reported to exert many pharmacological effects, including neuroprotection and anti-neuroinflammation, in PD. Dr. Chen's group reported that Rg1-regulated neuroinflammation may be associated with the inhibition of $M 1$ polarization in microglial cells [31].

Dopamine replacement therapy remains the major drug treatment for motor symptoms in PD. In addition to levo-DOPA treatment, numerous efforts have been made to develop DA receptor subtype selective ligands. Recently, some progress was made in developing multifunctional drugs, such as drugs that can modulate both dopaminergic function and cortical glutamate functions, e.g., drugs acting as dual D2/5-HT1A receptor agonists and D2/D3 agonists with antioxidant or iron-chelating activities $[8,9,32,33]$. Dr. Ye has updated the current progress in antiparkinsonian drugs [34].

Another pathological hallmark of PD is the formation of Lewy bodies (LBs), which contain misfolded and aggregated a-syn. As the Lewy body appears at an early stage of PD, detection of a-syn aggregation would be suitable for early diagnosis in the prodromal period of PD. The development of a-syn imaging probes will therefore not only be important for early diagnosis but may also provide early intervention opportunities for PD [35].

Xue-chu Zhen ${ }^{1}$ and Hong-Yuan $\mathrm{Chu}^{2}$ ${ }^{1}$ Jiangsu Key Laboratory of Neuropsychiatric Diseases and College of Pharmaceutical Sciences, Soochow University, 215123 Suzhou, China and ${ }^{2}$ Center For Neurodegenerative Science, Van Andel Institute,

Grand Rapids, MI, USA

Correspondence: Xue-chu Zhen (zhenxuechu@suda.edu.cn) or HongYuan Chu (hongyuan.chu@vai.org)

\section{ADDITIONAL INFORMATION}

Competing interests: The authors declare no competing interests.

\section{REFERENCES}

1. Kalia LV, Lang AE. Parkinson's disease. Lancet 2015;386:896-912.

2. Ascherio A, Schwarzschild MA. The epidemiology of Parkinson's disease: risk factors and prevention. Lancet Neurol 2016;15:1257-72.

3. Mazurskyy A, Howitt J. Initiation and Transmission of a-Synuclein Pathology in Parkinson's Disease. Neurochem Res. 2019. https://doi.org/10.1007/s11064-01902896-0.

4. Xu L, He D, Bai Y. Microglia-mediated inflammation and neurodegenerative disease. Mol Neurobiol 2016;53:6709-15.

5. Rani L, Mondal AC. Emerging concepts of mitochondrial dysfunction in Parkinson's disease progression: pathogenic and therapeutic implications. Mitochondrion 2020;50:25-34.

6. Dawson VL, Dawson TM. Promising disease-modifying therapies for Parkinson's disease. Sci Transl Med 2019;11:eaba1659.

7. Stoddard-Bennett T, Pera RR. Stem cell therapy for Parkinson's disease: safety and modeling. Neural Regen Res 2020;15:36-40.

8. Zheng J, Zhang X, Zhen X. Development of adenosine $A_{2 A}$ receptor antagonists for the treatment of parkinson's disease: a recent update and challenge. ACS Chem Neurosci. 2019;10:783-91.

9. Pinna A. Adenosine a2a receptor antagonists in parkinson's disease: Progress in clinical trials from the newly approved istradefylline to drugs in early development and those already discontinued. CNS Drugs 2014;28:455-74.

10. Przedborski S, Levivier M, Jiang H, Ferreira M, Jackson-Lewis V, Donaldson D, et al. Dose-dependent lesions of the dopaminergic nigrostriatal pathway induced by intrastriatal injection of 6-hydroxydopamine. Neuroscience 1995;67:631-47.

11. Javitch JA, D'Amato RJ, Strittmatter SM, Snyder SH. Parkinsonism-inducing neurotoxin, N-methyl-4-phenyl-1,2,3,6 -tetrahydropyridine: uptake of the metabolite $\mathrm{N}$-methyl-4-phenylpyridine by dopamine neurons explains selective toxicity. Proc Natl Acad Sci USA 1985;82:2173-7.

12. Moore DJ, Dawson TM. Value of genetic models in understanding the cause and mechanisms of Parkinson's disease. Curr Neurol Neurosci Rep. 2008;8:288-96.

13. Kin K, Yasuhara T, Kameda M, Date I. Animal models for Parkinson's disease research: trends in the 2000s. Int J Mol Sci. 2019;20:E5402.

14. Guo $\mathrm{CH}$, Cao T, Zheng LT, Waddington JL, Zhen XC. Development and characterization of an inducible Dicer conditional knockout mice model for Parkinson's Disease: validation of the antiparkinsonian effects of sigma-1 receptor agonist and dihydromyricetin. Acta Pharmacol Sin. 2020;41:499-507. https://doi. org/10.1038/s41401-020-0379-5.

15. Sheng J, Xu Z. Three decades of research on angiogenin: a review and perspective. Acta Biochim Biophys Sin. 2016;48:399-410.

16. Steidinger TU, Standaert DG, Yacoubian TA. A neuroprotective role for angiogenin in models of Parkinson's disease. J Neurochem. 2011;116:334-41.

17. Prehn JHM, Jirström E. Angiogenin and tRNA fragments in Parkinson's disease and neurodegeneration. Acta Pharmacol Sin. 2020;41:442-6. https://doi.org/ 10.1038/s41401-020-0375-9.

18. Galvan A, Wichmann T. Pathophysiology of parkinsonism. Clin Neurophysiol 2008;119:1459-74.

19. Lehmann J, Langer S. The striatal cholinergic interneuron: Synaptic target of dopaminergic terminals? Neuroscience. 1983;10:1105-20.

20. Chu HY. Synaptic and cellular plasticity in Parkinson's disease. Acta Pharmacol Sin. 2020;41:447-52. https://doi.org/10.1038/s41401-020-0371-0.

21. Smith Y, Bevan, Shink E, Bolam J. Microcircuitry of the direct and indirect pathways of the basal ganglia. Neuroscience. 1998;86:353-87.

22. Morris R, Martini DN, Madhyastha T, Kelly VE, Grabowski TJ, Nutt J, et al. Overview of the cholinergic contribution to gait, balance and falls in Parkinson's disease. Parkinsonism Relat Disord. 2019;63:20-30.

23. Liu C. Targeting cholinergic system in Parkinson's disease. Acta Pharmacol Sin. 2020;41:453-63. https://doi.org/10.1038/s41401-020-0380-z.

24. Fytche DH, Creese B, Politis M, Chaudhuri KR, Weintraub D, Ballard C, Aarsland D. The psychosis spectrum in Parkinsondisease. Nat Rev Neurol. 2017;13:81-95.

25. Waddington JL. Psychosis in Parkinson's disease and parkinsonism in antipsychotic-naive schizophrenia spectrum psychosis: clinical, nosological and pathobiological challenges. Acta Pharmacol Sin. 2020;41:464-70. https://doi.org/ 10.1038/s41401-020-0373-y.

26. Kusumi I, Boku S, Takahashi Y. Psychopharmacology of atypical antipsychotic drugs: From the receptor binding profile to neuroprotection and neurogenesis. Psychiatry Clin Neurosci. 2015;69:243-58.

27. Jiang L, Wu X, Wang S, Chen SH, Zhou H, Wilson B, et al. Clozapine metabolites protect dopaminergic neurons through inhibition of microglial NADPH oxidase. J Neuroinflammation 2016;13:110. 
28. Xiong YJ, Song YZ, Zhu Y, Zuo WQ, Zhao YF, Shen X, et al. Neuroprotective effects of olanzapine against rotenone-induced toxicity in PC12 cells. Acta Pharmacol Sin. 2020;41:508-15. https://doi.org/10.1038/s41401-020-0378-6.

29. Ren ZX, Zhao YF, Cao T, Zhen X. Dihydromyricetin protects neurons in an MPTPinduced model of Parkinson's disease by suppressing glycogen synthase kinase-3 beta activity. Acta Pharm Sin. 2016;37:1315-24

30. Yu ZR, Jia WH, Liu C, Wang HQ, Yang HG, He GR, et al. Ganoderic acid A protects neural cells against NO stress injury in vitro via stimulating $\beta$ adrenergic receptors. Acta Pharmacol Sin. 2020;41:516-22. https://doi.org/10.1038/s41401-0200356-z.

31. Liu JQ, Zhao M, Zhang Z, Cui LY, Zhou X, Zhang W, et al. Rg1 improves LPSinduced Parkinsonian symptoms in mice via inhibition of NF-KB signaling and modulation of M1/M2 polarization. Acta Pharmacol Sin. 2020;41:523-34. https:// doi.org/10.1038/s41401-020-0358-x.
32. Cheong SL, Federico S, Spalluto G, Klotz KN, Pastorin G. The current status of pharmacotherapy for the treatment of parkinson's disease: Transition from single-target to multitarget therapy. Drug Discov Today 2019;24:1769-83.

33. Zhang H, Ye N, Zhou S, Guo L, Zheng L, Liu Z, et al. Identification of $\mathrm{N}$-propylnoraporphin-11-yl 5-(1,2-dithiolan-3-yl)pentanoate as a new anti-Parkinson's agent possessing a Dopamine $\mathrm{D}(2)$ and Serotonin 5-HT(1A) dual-agonist profile. J Med Chem. 2011;54:4324-38.

34. Mao Q, Qin WZ, Zhang A, Ye N. Recent advances in dopaminergic strategies for the treatment of Parkinson's disease. Acta Pharmacol Sin. 2020;41:471-82. https://doi.org/10.1038/s41401-020-0365-y.

35. Xu MM, Ryan P, Rudrawar S, Quinn RJ, Zhang HY, Mellick GD. Advances in the development of imaging probes and aggregation inhibitors for alpha-synuclein. Acta Pharmacol Sin. 2020;41:483-98. https://doi.org/10.1038/s41401-019-0304-y. 